\title{
Factors Affecting Survival in Neuroendocrine Tumors: A 15-Year Single Center Experience
}

\author{
Abdullah Sakin ${ }^{1 *}$, Makbule Tambas², Saban Secmeler ${ }^{3}$, Orçun Can ${ }^{3}$, Serdar \\ Arici $^{3}$, Nurgul Yasar ${ }^{3}$, Caglayan Geredeli ${ }^{3}$, Cumhur Demir ${ }^{3}$, Sener Cihan ${ }^{3}$
}

\begin{abstract}
Background: Neuroendocrine tumors are a heterogeneous group of tumors that can originate from all of the neuroendocrine cells in the body, mostly from the gastrointestinal tract. In addition to early diagnosis, streaming patients into appropriate prognostic groups is an important component of treatment. In this study, we examined the factors that affect survival in patients we followed in our center between 2000-2016. Methods: The demographic data, clinical and pathological features of patients were obtained from their medical files. TNM staging and tumor grading were performed according to AJCC and WHO 2010 classification. SPSS 15.0 for Windows programme was used for statistical analysis. Results: 85 patients ( 32 male, 53 female) were included into the study. The median age of the patients was 55,7 (27-83) years. Eighty percent of the tumors were of gastroenteropancreatic system, most commonly stomach $(27.1 \%)$ origin. Nineteen patients $(22.4 \%)$ died during follow-up. In univariate analysis; age $(p<0,001)$, stage $(p=0.002)$, primary tumor localization $(p=0.005)$, grade $(p<0.001)$, Ki-67 value $(p<0.001)$, number of metastases $(p=0.001)$ and type of surgery $(p<0.001)$ were found to be factors affecting survival. Age $(p=0.024)$ and Ki67 $(p<0.001)$ were the independent prognostic factors for survival in multivariate analysis. For the cut-off value of $6 \%$, Ki- 67 had a sensitivity of $83.3 \%$ and specifity of $71.4 \%$ for survival determination. Conclusion: Ki-67 ratio and age were the most important factors affecting survival in neuroendocrine tumors in our study. Ki-67 ratio has a high sensitivity and specificity for predicting survival, a cut-off value of $6 \%$ may be used to predict survival.
\end{abstract}

Keywords: Age- grade- Ki-67- neuroendocrine tumor- survival

Asian Pac J Cancer Prev, 19 (12), 3597-3603

\section{Introduction}

Neuroendocrine tumors (NET) are a heterogeneous group of tumors that can originate from all of the neuroendocrine cells in the body, mostly from the lung and gastrointestinal tract including stomach, pancreas, small and large intestine, rectum. They can occur at any age, although it is often seen over 50 years. The incidence of NET is higher in men than in women. Even though they usually exhibit indolent clinical course, they may become very aggressive and rapidly become metastatic. Since most of NET are not functional, they often cause no signs and symptoms, which makes early diagnosis difficult and decreases survival by reducing the chance of curative treatment (Yao et al., 2008). In addition to early diagnosis, streaming patients into appropriate prognostic groups is an important component of treatment. However, the absence of frequently accepted classifications limits its benefit on survival (Bilimoria et al., 2007).

There is insufficient information about the incidence and frequency of many NET subgroups, including those with unknown primaries. In addition, long-term follow-up and survival-related data are limited in NET patients. The survival and the factors affecting it in patients with NET in many countries have not been identified. This suggests that further studies on prognostic parameters are needed (Oh et al., 2012).

In this study, we evaluated the prognostic significance of the clinicopathologic parameters routinely used in daily practise and the treatments administrated to the NET patients that we followed in our center between 2000 and 2016.

\section{Materials and Methods}

Patients who were diagnosed with pathologically verified NET and treated and followed up at our clinic between 2000 and 2016 were included in the study. The data concerning patients' age, gender, complaint for hospital admission, smoking history, the presence

${ }^{1}$ Department of Medical Oncology, Yuzuncu Yil University Medical School, 65090, Van, ${ }^{2}$ Department of Radiation Oncology, ${ }^{3}$ Department of Medical Oncology, University of Health Sciences, Okmeydani Training and Research Hospital, 34384, Istanbul, Turkey. *For Correspondence: Drsakin@hotmail.com 
of carcinoid syndrome, stage, location of the primary, the location and number of metastases, type of surgery and treatment applied were obtained from their medical files. Patients with incomplete data, missing data, or multiple primers were excluded from the study. A total of 85 patients ( 32 males and 53 females) were included in the study. The TNM staging of patients and grading $(\mathrm{G})$ of the tumor were performed according to AJCC and 2010 WHO classification, respectively (Bosman, 2010); World Health Organization; International Agency for Research on Cancer. WHO Classification of Tumours of the Digestive System. 4th ed. Lyon: International Agency for Research on Cancer).

The study protocol was approved by the Corporate Ethics Committee and found to comply with ethical principles for epidemiological investigations.

SPSS 15.0 for Windows program was used for statistical analysis. Descriptive statistics were given as mean, standard deviation, minimum, maximum for numerical variables, number and percentage for categorical variables. The numerical variables in the independent two groups were analyzed by Student $t$ test and Mann Whitney U test if normal distribution condition was provided and not met, respectively. The comparisons of ratios between groups were made with Chi Square Analysis. Monte Carlo simulation was applied when conditions were not met. The survival analyzes were performed with Kaplan Meier Analysis. Determinants for survival were examined by Cox Regression Analysis. In univariate analysis, forward stepwise model was used for values with $\mathrm{p}<0.100$. The cut-off values were determined by using Roc Curve Analysis. The statistical significance level of alpha was accepted as $\mathrm{p}<0.05$.

\section{Results}

A total of 85 patients, 32 (37.6\%) male and 53 (62.4\%) female, were included in the study. The median age was 55.7 (27-83) years. Eighty percent of the tumors were of gastroenteropancreatic system, most commonly stomach $(27.1 \%)$ origin.

According to WHO classification, well differentiated NET (G1), moderately differentiated NET (G2) and neuroendocrine carcinoma $(\mathrm{NEC})(\mathrm{G} 3)$ were detected in 53 (62.1\%), 6 (7.1\%), $26(30.8 \%)$ patients, respectively. According to the AJCC / UICC staging, 31 (36.5\%) patients had stage $1 ; 6(7.1 \%)$ patients had stage $2 ; 11$ (12.9\%) patients had stage $3 ; 37(43.5 \%)$ patients had stage 4 disease. Of the $59(69.4 \%)$ patients, 54 (63.5\%) and $5(5.9 \%)$ underwent curative and palliative surgery, respectively. The lymph node metastasis was detected in $18(30.5 \%)$ patients. At the time of diagnosis, $44.8 \%$ of patients had distant metastases (Table 1).

The somatostatin analogs to $25(36.8 \%)$ patients, metastatic first-line cytotoxic chemotherapy (CT) to 36 (44.1\%) patients, and second line CT to $6(7.4 \%)$ patients were given as systemic treatment. $3(3.5 \%)$ patients received everolimus. Two patients $(2.3 \%)$ received peptide receptor radionuclide therapy (PRRT) (Table 1).

It was observed that the tumor grades varied with the localization of the tumors. Statistically significant
Table 1. Demographic Data, Applied Treatments and Response Rates

\begin{tabular}{|c|c|c|c|}
\hline & & Mean \pm SD & Min-Max \\
\hline \multicolumn{2}{|l|}{ Age } & $55.7 \pm 14.4$ & $27-83$ \\
\hline \multicolumn{2}{|l|}{ Ki-67 ratio $(\%)$} & $15.4 \pm 23.1$ & Jan-95 \\
\hline \multicolumn{2}{|l|}{ Mitosis number } & $3.8 \pm 9.6$ & $0-50$ \\
\hline \multicolumn{2}{|l|}{ Number of metastases } & $0.87 \pm 1.12$ & $0-3$ \\
\hline & & $\mathrm{n}$ & $\%$ \\
\hline \multirow[t]{2}{*}{ Gender } & Male & 32 & 37.6 \\
\hline & Female & 53 & 62.4 \\
\hline \multicolumn{2}{|l|}{ Smoking } & 30 & 35.3 \\
\hline \multirow[t]{11}{*}{ Symptom } & Abdominal pain & 54 & 63.5 \\
\hline & GIS hemorage & 8 & 9.4 \\
\hline & Weight loss & 5 & 5.9 \\
\hline & Symptomatic & 5 & 5.9 \\
\hline & Hot flushes & 3 & 3.5 \\
\hline & Back pain & 2 & 2.4 \\
\hline & Shortness of breath & 3 & 3.5 \\
\hline & Swallowing difficulty & 2 & 2.4 \\
\hline & Jaundice & 1 & 1.2 \\
\hline & Palpable mass & 1 & 1.2 \\
\hline & Diarrhea & 1 & 1.2 \\
\hline \multicolumn{2}{|l|}{ Carcinoid Syndrome } & 7 & 8.2 \\
\hline \multirow[t]{3}{*}{ Grade } & G1 & 53 & 62.1 \\
\hline & $\mathrm{G} 2$ & 6 & 7.1 \\
\hline & G3 & 26 & 30.8 \\
\hline \multirow[t]{4}{*}{ Stage } & I & 31 & 36.5 \\
\hline & II & 6 & 7.1 \\
\hline & III & 11 & 12.9 \\
\hline & IV & 37 & 43.5 \\
\hline \multirow[t]{7}{*}{ Primary Localization } & Stomach & 23 & 27.1 \\
\hline & Pancreas & 18 & 21.2 \\
\hline & Small bowel & 10 & 11.8 \\
\hline & Appendix & 10 & 11.8 \\
\hline & Unknown primary & 9 & 10.6 \\
\hline & Lung & 8 & 9.4 \\
\hline & Colorectal & 7 & 8.2 \\
\hline \multirow[t]{4}{*}{ Metastases } & None & 47 & 55.3 \\
\hline & Liver & 34 & 40 \\
\hline & Lung & 2 & 2.4 \\
\hline & Bone & 2 & 2.4 \\
\hline \multirow{2}{*}{$\begin{array}{l}\text { Lymph Node } \\
\text { Metastases }\end{array}$} & No & 41 & 69.5 \\
\hline & Yes & 18 & 30.5 \\
\hline \multirow[t]{3}{*}{ Surgery Type } & None & 26 & 30.6 \\
\hline & Curative & 54 & 63.5 \\
\hline & Palliative & 5 & 5.9 \\
\hline \multicolumn{2}{|l|}{ Octreotide } & 25 & 36.8 \\
\hline \multirow[t]{3}{*}{ Octreotide response } & PR & 10 & 14.7 \\
\hline & SD & 13 & 19.1 \\
\hline & PD & 2 & 2.9 \\
\hline \multicolumn{2}{|l|}{ Everolimus } & 3 & 4.4 \\
\hline \multirow[t]{2}{*}{ Everolimus response } & PR & 1 & 1.5 \\
\hline & SD & 2 & 2.9 \\
\hline
\end{tabular}


Table 1. Continued

\begin{tabular}{lccc}
\hline & & Mean \pm SD & Min-Max \\
\hline 1. line CT & & 36 & 44.1 \\
1.line CT response & CR & 6 & 8.8 \\
& PR & 18 & 17.6 \\
& SD & 7 & 10.3 \\
2. line CT & PD & 5 & 7.4 \\
2.line CT response & PR & 6 & 7.4 \\
3. line CT & SD & 4 & 5.9 \\
3.line CT response & SD & 2 & 1.5 \\
& PD & 3 & 4.4 \\
PRRT & & 2 & 2.9 \\
\hline
\end{tabular}

G1, well differentiated; G2, moderately differentiated; G3, neuroendocrine carcinoma; CR, complete response; SD, Stable disease; PR, Partial response; PD, Progressive disease; PRRT: Peptide receptor radionuclide therapy

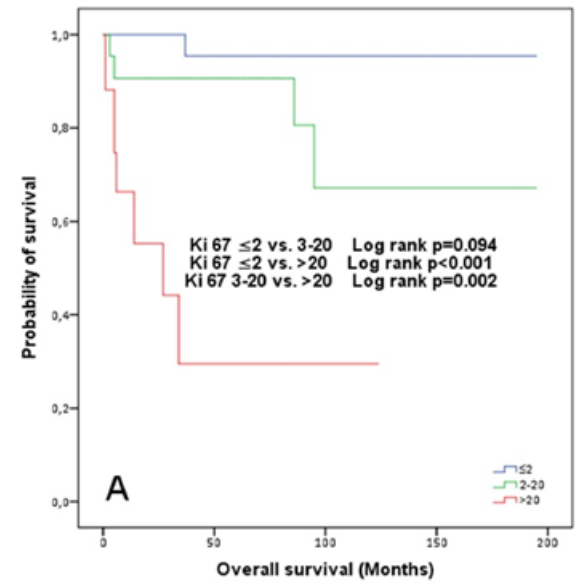

differences were found in tumor grades of patients with primary lung $(\mathrm{p}=0.001)$, stomach $(\mathrm{p}=0.0001)$, and pancreas $(p=0.022)$ compared to the others. G2 ratio of patients with primary lung, G3 ratio of patients with primary stomach and G1 ratio of patients with primary pancreas were significantly higher than the other primaries (Table 3).

During follow-up, 19 (22.4\%) patients died. 5-, 10-, and 15 -year survival rates of all patients were $75.2 \%$, $67.8 \%$, and $60.3 \%$, respectively; while median survival time was not reached. According to gradings, 5-,10- and 15 -year survival rates were found as $95 \%, 95 \%$, and $95 \%$ for $\mathrm{G} 1 ; 91.1 \%, 67.5 \%$, and $67.5 \%$ for $\mathrm{G} 2 ; 19.1 \%, 19.1 \%$, and $19.1 \%$ for G3; respectively; According to stages, $5-, 10$ - and 15-year survival rates were found as $97.1 \%$, $93.3 \%$, and $93.3 \%$ for stage I+II; $88.3 \%, 74.1 \%$, and $55.0 \%$ for stage III; $57.4 \%, 38.3 \%$, and $38.3 \%$ for stage $\mathrm{IV}$; respectively (Table 2).

Age $(p=0.007), K i-67$ ratio $(p=0.001)$, number of

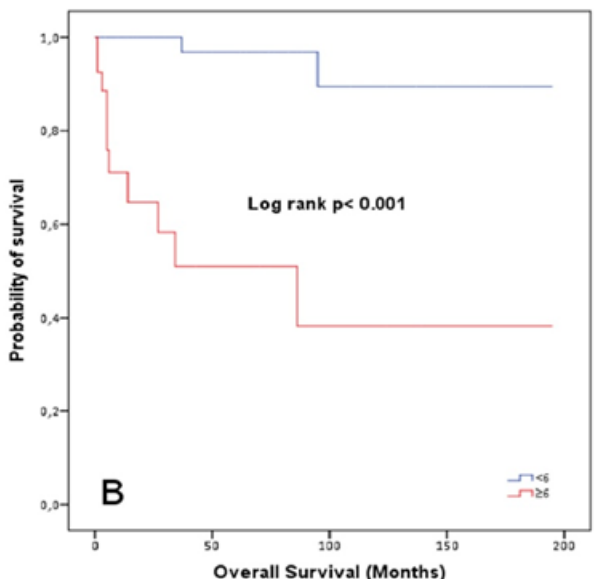

Figure 1. Survival by Ki-67 Groups

Table 2. Primary Tumor Localizations According to Grades

\begin{tabular}{|c|c|c|c|c|c|c|c|}
\hline \multirow[t]{2}{*}{ Primary Localization } & \multicolumn{2}{|c|}{$\mathrm{G} 1(\mathrm{n}=53)$} & \multicolumn{2}{|c|}{$\mathrm{G} 2(\mathrm{n}=6)$} & \multicolumn{2}{|c|}{$\mathrm{G} 3(\mathrm{n}=26)$} & \multirow[b]{2}{*}{$\mathrm{p}$} \\
\hline & $\mathrm{n}$ & $\%$ & $\mathrm{n}$ & $\%$ & $\mathrm{n}$ & $\%$ & \\
\hline Lung & 5 & 9.4 & 3 & 50 & 0 & 0 & 0.001 \\
\hline Stomach & 7 & 13.2 & 0 & 0 & 16 & 61.8 & 0.001 \\
\hline Pancreas & 16 & 30.2 & 1 & 16.7 & 1 & 3.8 & 0.022 \\
\hline Small bowel & 7 & 13.2 & 2 & 33.3 & 1 & 3.8 & 0.075 \\
\hline Colorectal & 3 & 5.7 & 0 & 0 & 4 & 15.3 & 0.627 \\
\hline Appendix & 10 & 18.9 & 0 & 0 & 0 & 0 & 0.164 \\
\hline Unknown & 5 & 9.4 & 0 & 0 & 4 & 15.3 & 1 \\
\hline Overall Survival (Years) & $\mathrm{G} 1 \%(\%)$ & & $\mathrm{G} 2(\%)$ & & G1(\%) & & Total $(\%)$ \\
\hline 5 & 95.5 & & 91.1 & & 19.1 & & 75.2 \\
\hline 10 & 95.5 & & 67.5 & & 19.1 & & 67.8 \\
\hline 15 & 95.5 & & 67.5 & & 19.1 & & 60.3 \\
\hline Stage (Years) & $\mathrm{I}+\mathrm{II}(\mathrm{n}=37)$ & & III $(n=11)$ & & IV $(n=37)$ & & $\mathrm{p}$ \\
\hline 5 & 97.1 & & 88.3 & & 57.4 & & 0.001 \\
\hline 10 & 93.3 & & 74.1 & & 38.3 & & 0.001 \\
\hline 15 & 93.3 & & 0.55 & & 38.3 & & 0.001 \\
\hline
\end{tabular}

G1, well differentiated; G2, moderately differentiated; G3, neuroendocrine carcinoma 
mitosis $(p=0.031)$, number of metastases $(p=0.016)$, the ratio of gastric localization of the primary tumor $(p=0.001)$, presence of distant metastases $(p=0.028)$, presence of lymph node metastases $(p=0.049)$ and CT utilization rates $(p=0.001)$ were statistically significantly higher in patients with exitus compared with those alive. In addition, the rates of $\mathrm{G} 3(\mathrm{p}<0.001)$ and stage IV disease (0.016) were significantly higher, whereas curative surgery rate was significantly lower $(\mathrm{p}=0.007)$ in patients who died (Table 3).

In univariate analysis; age $(p<0.001)$, stage $(p=0.002)$, primary tumor localization $(\mathrm{p}=0.005)$, grade $(\mathrm{p}<0.001)$, Ki-67 ratio $(p<0.001)$, the number of metastasis $(p=0.001)$ and the type of surgery ( $<<0.001)$ were found to be the factors affecting survival (Table 4 ). When factors affecting the OS were evaluated, age $(p=0.024)$ and $\mathrm{Ki}-67$ ratio $(p<0.001)$ were found to be the most significant factors according to Forward Stepwise analysis based on model consisted of variables of which $\mathrm{p}$ values were determined as $<0.100$ in univariate analysis (age, smoking, primary tumor localization, grade, stage, metastasis, type of surgery, Ki-67 ratio) (Table 5).

There was a statistically significant difference in survival rates in the Ki-67 ratio groups $(\mathrm{p}<0.001)$. Patients with a Ki-67 ratio of $>20 \%$ had a statistically significant lower survival rate than those with $\leq 2 \%(p<0.001)$ and $3-20 \%(p=0.002)$. On the orher hand, no significant difference in survival rates was detected between patients with Ki-67 value of $\leq 2 \%$ and $3-20 \%(p=0.094)$ (Figure 1). The median survival was $27 \pm 13.2$ (95\% CI:1.0-52.5)

Table 3. Comparison of Characteristics of Patients with and without Exitus

\begin{tabular}{|c|c|c|c|c|c|c|}
\hline & & \multicolumn{3}{|c|}{ Last status } & & \multirow{3}{*}{$\mathrm{p}$} \\
\hline & & Exitus & & Alive & & \\
\hline & & Mean \pm SD & & Mean \pm SD & & \\
\hline Age & & $63.4 \pm 12.2$ & & $52.9 \pm 14.3$ & & 0.007 \\
\hline Ki-67 ratio (\&) & & $37.3 \pm 31.3$ & & $9.1 \pm 15.6$ & & 0.001 \\
\hline Mitosis number & & $18.3 \pm 22.9$ & & $1.5 \pm 1.5$ & & 0.031 \\
\hline \multirow[t]{2}{*}{ Metastases number } & & $1.39 \pm 1.24$ & & $0.68 \pm 1.02$ & & 0.016 \\
\hline & & $\mathrm{n}$ & $\%$ & $\mathrm{n}$ & $\%$ & $\mathrm{p}$ \\
\hline \multirow[t]{2}{*}{ Gender } & Female & 12 & 63.2 & 41 & 62.1 & 0.891 \\
\hline & Male & 7 & 36.8 & 25 & 37.9 & \\
\hline Carcinoid Syndrome & & 0 & 0 & 7 & 10.6 & 0.341 \\
\hline Smoking & & 10 & 52.6 & 20 & 30.3 & 0.073 \\
\hline \multirow[t]{7}{*}{ Primary localization } & Lung & 0 & 0 & 8 & 12.1 & 0.315 \\
\hline & Stomach & 12 & 63.2 & 12 & 18.2 & 0.001 \\
\hline & Pancreas & 2 & 10.5 & 16 & 24.2 & 0.323 \\
\hline & Small bowel & 1 & 5.3 & 9 & 13.6 & 0.666 \\
\hline & Colorectal & 2 & 10.5 & 5 & 7.6 & 0.652 \\
\hline & Appendix & 1 & 5.3 & 9 & 13.6 & 1 \\
\hline & Unknown & 1 & 5.3 & 7 & 10.6 & 0.666 \\
\hline \multirow[t]{3}{*}{ Grade } & G1 & 4 & 21.1 & 49 & 74.4 & $<0.001$ \\
\hline & G2 & 1 & 5.3 & 5 & 7.7 & \\
\hline & G3 & 14 & 73.7 & 12 & 17.9 & \\
\hline \multirow[t]{4}{*}{ Stage } & I & 1 & 5.3 & 30 & 45.5 & 0.016 \\
\hline & II & 1 & 5.3 & 5 & 7.6 & \\
\hline & III & 3 & 15.8 & 8 & 12.1 & \\
\hline & IV & 14 & 73.7 & 23 & 34.8 & \\
\hline Metastases & & 14 & 73.7 & 24 & 36.3 & 0.028 \\
\hline Lymph Node Metastases & & 5 & 66.7 & 13 & 28 & 0.049 \\
\hline \multirow[t]{3}{*}{ Cerrahi Tipi } & None & 11 & 57.9 & 15 & 22.7 & 0.007 \\
\hline & Curative & 5 & 26.3 & 49 & 74.2 & \\
\hline & Palliative & 3 & 15.8 & 2 & 3 & \\
\hline Octreotie & & 6 & 33.3 & 19 & 38 & 0.725 \\
\hline Everolimus & & 2 & 11.1 & 1 & 2 & 0.169 \\
\hline 1.line CT & & 16 & 84.2 & 20 & 30.3 & 0.001 \\
\hline 2.line CT & & 2 & 13.3 & 4 & 20 & 0.111 \\
\hline 3.line CT & & 1 & 5.6 & 2 & 4 & 1 \\
\hline
\end{tabular}

G1, well differentiated; G2, moderately differentiated; G3, neuroendocrine carcinoma; CT, chemotherapy 


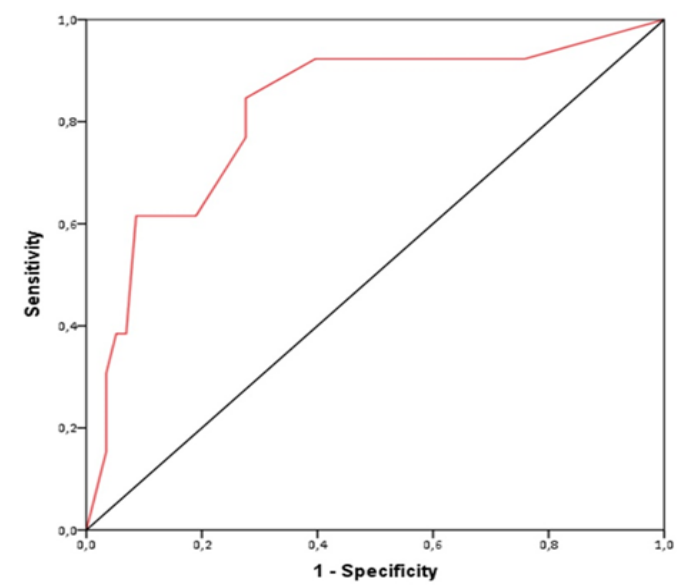

Figure 2. Receiver Operating Characteristic Curve Analyses for the Determination of Mortality, the Sensitivity and Specificity of the Ki-67 Ratio were Found as $83.3 \%$ and $71.4 \%$ for the cut-off value of $>6 \%$ (AUC:0.813 (\%95 CI: 0.664-0.963).

Table 4. Factors Affecting Survival in Univariate Analysis

\begin{tabular}{lc}
\hline Variables & Log rank $\mathrm{p}$ \\
\hline Gender & 0.791 \\
Age $(<65 \mathrm{vs} \geq 65)$ & $<0.001$ \\
Carcinoid Syndrome presence & 0.13 \\
Smoking & 0.085 \\
Stage & 0.002 \\
Grade & $<0.001$ \\
Ki-67 \% $(\leq 2.2-20 .>20)$ & $<0.001$ \\
Primary tumor localization & 0.005 \\
Metastases number & 0.001 \\
Curative surgery & $<0.001$ \\
Lymph node metastases & 0.986 \\
\hline
\end{tabular}

months in the Ki67 $>20 \%$ group, whereas median survival was not achieved in the other groups.

According to the ROC analysis for the determination of mortality, the sensitivity and specificity of the Ki-67 ratio were found as $83.3 \%$ and $71.4 \%$ for the cut-off value of $>6 \%$ (AUC:0.813 (\%95 CI: 0.664-0.963) (Figure 2). The cumulative survival rate of patients with a $\mathrm{Ki} 67$ ratio of $\geq 6 \%$ was found to be statistically significantly lower than those with a Ki67 ratio of $<6 \%(\mathrm{p}<0.001)$ (Figure 1$)$. The median survival was not reached in the group with a Ki67 ratio of $<6 \%$ whereas it was $86 \pm 41.9$ (95\% CI:3.8-168.1) months in the group with a Ki67 ratio of $\geq 6 \%$.

\section{Discussion}

The naming and classification of NET have been

Table 5. Survival Determining Factors in Multivariate Analysis

\begin{tabular}{lcccc}
\hline & $\mathrm{p}$ & $\mathrm{HR}$ & \%95 CI & \\
\hline Age (Years) & 0.024 & 1.067 & 1.009 & 1.128 \\
Ki67 \% & $<0.001$ & 1.048 & 1.024 & 1.073 \\
\hline
\end{tabular}

changed several times, making it difficult to collect epidemiological information and compare studies published in the literature. The actual incidence of NETs is not known due to the lack of sufficient multicentric and epidemiological studies. This may explain the difference in incidence of NET between gender, race, country and continent (Hauso et al., 2008).

Over the last decade, attempts have been made to develop existing classification systems. There is limited data on long-term follow-up and survival in patients with NET. Because of infrequency and the differences in the diagnosis of NET, it is difficult to identify high risk factors. There are only a few studies that define prognostic factors, thus, factors affecting survival of patients with NET is lacking in many countries (Faggiano et al., 2012).

The median age of the patients at our study was 55.7 years, similar to other studies (Niederle et al., 2010; Araujo et al., 2013; Lewkowicz et al., 2015; Nikou et al., 2016). Five percent of the cases were asymptomatic. The incidence of carcinoid syndrome was $8.2 \%$. Similar to other studies, the most common symptom was abdominal pain (Araujo et al., 2013; Lewkowicz et al., 2015). The most common disease grade seen in our study was G1. The most common localizations of the G1 disease were of the rectum and appendix in other studies, whereas it was of pancreas and appendix in our study (Niederle et al., 2010; Lewkowicz et al., 2015).

The pancreas and lung were the most common primary localizations in the study by Nikou et al., (2016). In another study, the most common primary localizations were alined as rectum, duodenum, pancreas and stomach while the most frequent stage, grade and metastatic site were stage 1, grade 1 and the liver, respectively (Lim et al., 2017). In our study, unlike other studies, the most common localizations were stomach, pancreas and small bowel (Garcia-Carbonero et al., 2010; Niederle et al., 2010; Lim et al., 2011; Lewkowicz et al., 2015). The most common distant metastasis site was liver.

Grade and $\mathrm{Ki}-67$ ratio are required for pathologic classification and have prognostic significance. The $\mathrm{Ki} 67$ ratio was found to be $<2 \%$ in most of the studies (Garcia-Carbonero et al., 2010; Niederle et al., 2010; Lim et al., 2011; Araujo et al., 2013; Lewkowicz et al., 2015; Nikou et al., 2016; Lim et al., 2017). Likewise, in our study, the Ki67 rate was found to be $\leq 2 \%$ in $63.1 \%$, $3-20 \%$ in $7.1 \%$, and $>20 \%$ in $25 \%$ of patients.

The only curative treatment method in NET is the surgical resection. Surgery should be considered in patients with early stage, locoregional and resectable metastatic disease (Bilimoria et al., 2007). In our study, curative surgery was applied to $63.5 \%$ of patients and lymph node metastasis was detected in $30.5 \%$ of patients who underwent surgical treatment. Of our patients, $44.8 \%$ were metastatic at the time of diagnosis.

Somatostatin analogues in patients with NET provide symptom control, improve quality of life and control disease progression. Somatostatin analogues are a recommended treatment option for nonfunctional and functional G1/G2 NETs (Saglam et al., 2015). In our study, somatostatin analogues were given to $25(36.8 \%)$ patients. Partial response in $10(14.7 \%)$ patients, stable disease in 
$13(19.1 \%)$ patients and progressive disease in $2(2.9 \%)$ patients were observed. Due to tumor burden, the first line CT (platinum+etoposide) was given to $36(44.1 \%)$ patients with metastatic disease. Of those, $6(8.8 \%)$ patients had complete response, $18(17.6 \%)$ patients had partial response and $7(10.3 \%)$ patients had stable disease. In addition, 6 (7.4\%) patients were given second line CT while PRRT was administered to 2 patients.

5 -year overall survival rate varies between $67-90 \%$ in studies (Lim et al., 2011; Lewkowicz et al., 2015; Lim et al., 2017; Ma et al., 2017). In the study by Ma et al., (2017) the 5-year survival rate was $58.4 \%$ while survival rates in $\mathrm{G} 1, \mathrm{G} 2$ and $\mathrm{G} 3$ were $100 \%, 71.4 \%$ and $44.4 \%$, respectively. In our study, 5-, 10-, and 15-year survival rates were $75.2 \%, 67.8 \%$, and $60.3 \%$, respectively. The $5-, 10$ - and 15 -year survival rates were estimated as $95 \%$, $95 \%$, and $95 \%$ for $\mathrm{G} 1 ; 91.1 \%, 67.5 \%, 67.5 \%$ for $\mathrm{G} 2$; $19.1 \%, 19.1 \%$, and $19.1 \%$ for G3; respectively. The decrease from 5- to 10-year survival rates of patients with G2 NET was remerkable which suggests that attention should be paid to the late recurrences after 5 years in G2 disease.

In a study that evaluated prognostic factors after resection of pancreatic NET, the presence of tumor necrosis, lymph node and liver metastasis was found to be associated with disease-free survival whereas age, tumor grade and the presence of distant metastasis were detected as the most significant determinants of survival (Bilimoria et al., 2008). Various studies have reported different survival rates according to tumor localization (Garcia-Carbonero et al., 2010; Lim et al., 2011; Lewkowicz et al., 2015). In the study by Lewkowicz et al., (2015) advanced stage, G2 and presence of metastasis at diagnosis were determined to be associated with poor prognosis in the univariate analysis, while presence of advanced stage and metastasis was found as the independent risk factors for poor outcome in the multivariate analysis. In another study, grade and stage were found as the independent risk factors for survival (Garcia-Carbonero et al., 2010). In the study by Ma et al., (2017) it was determined that 5-year survival rate of patients with advanced age, tumor localized in stomach, duodenum and colon, a tumor size of $\geq 4 \mathrm{~cm}$ and G3 disease was lower in univariate analysis. In multivariate analysis, age, stage, lymph node and distant metastasis were found to be independent risk factors affecting the prognosis of patients. In our study, age, Ki-67 and mitosis rate, stage, gastric localization, presence of distant metastases at the time of diagnosis, number of metastases, presence of lymph node metastasis and CT use were found to be factors affecting survival in univariate analysis. In multivariate analysis, age and $\mathrm{Ki}-67$ ratio were found to be the most significant factors. The lower survival rates in gastric localization may be due to higher values of Ki-67 ratios of tumors in gastric localization. Furthermore, the use of $\mathrm{CT}$ in the treatment of symptomatic patients with high tumor burden may explain the lower survival rates in this group.

As a conclusion, in our study, age, Ki-67 ratio, number of mitosis, number of metastases, gastric localization of the primary tumor, presence of distant metastases, presence of lymph node metastases, G3 and stage IV disease and CT utilization rates were statistically significant higher in patients with exitus compared with those alive. The Ki-67 ratio and age were determined as the most important factors affecting survival. Ki-67 ratio has high sensitivity and specificity in predicting survival. We think that the Ki-67 ratio of $\geq 6 \%$ might be used to estimate survival.

\section{Funding Source}

None.

\section{Conflict of Interest}

The authors declare that they have no conflict of interest.

\section{References}

Araujo PB, Cheng S, Mete O, et al (2013). Evaluation of the WHO 2010 grading and AJCC/UICC staging systems in prognostic behavior of intestinal neuroendocrine tumors. PLoS One, 8, e61538.

Bilimoria KY, Bentrem DJ, Merkow RP, et al (2007). Application of the pancreatic adenocarcinoma staging system to pancreatic neuroendocrine tumors. J Am Coll Surg, 205, 558-63.

Bilimoria KY, Talamonti MS, Tomlinson JS, et al (2008). Prognostic score predicting survival after resection of pancreatic neuroendocrine tumors: analysis of 3851 patients. Ann Surg, 247, 490-500.

Bosman FT (2010). World Health Organization; International Agency for Research on Cancer. WHO Classification of Tumours of the Digestive System. 4th ed. Lyon: International Agency for Research on Cancer.

Faggiano A, Ferolla P, Grimaldi F, et al (2012). Natural history of gastro-entero-pancreatic and thoracic neuroendocrine tumors. Data from a large prospective and retrospective Italian epidemiological study: the NET management study. $J$ Endocrinol Invest, 35, 817-23.

Garcia-Carbonero R, Capdevila J, Crespo-Herrero G, et al (2010). Incidence, patterns of care and prognostic factors for outcome of gastroenteropancreatic neuroendocrine tumors (GEP-NETs): results from the National Cancer Registry of Spain (RGETNE). Ann Oncol, 21, 1794-803.

Hauso O, Gustafsson BI, Kidd M, et al (2008). Neuroendocrine tumor epidemiology: contrasting Norway and North America. Cancer, 113, 2655-64.

Lewkowicz E, Trofimiuk-Muldner M, Wysocka K, et al (2015). Gastroenteropancreatic neuroendocrine neoplasms: a 10-year experience of a single center. Pol Arch Med Wewn, 125, 337-46.

Lim CH, Lee IS, Jun BY, et al (2017). Incidence and clinical characteristics of gastroenteropancreatic neuroendocrine tumor in Korea: a single-center experience. Korean J Intern Med, 32, 452-8.

Lim T, Lee J, Kim JJ, et al (2011). Gastroenteropancreatic neuroendocrine tumors: incidence and treatment outcome in a single institution in Korea. Asia Pac J Clin Oncol, 7, 293-9.

Ma X, Zhao W, Zhuang C, et al (2017). Clinicopathological classification and prognostic factors of gastrointestinal neuroendocrine neoplasms: an analysis of 119 cases. Zhonghua Wei Chang Wai Ke Za Zhi, 20, 997-1001.

Niederle MB, Hackl M, Kaserer K, et al (2010). Gastroenteropancreatic neuroendocrine tumours: the current incidence and staging based on the WHO and European Neuroendocrine Tumour Society classification: an analysis 
based on prospectively collected parameters. Endocr Relat Cancer, 17, 909-18.

Nikou GC, Pazaitou-Panayiotou K, Dimitroulopoulos D, et al (2016). Results of a prospective multicenter neuroendocrine tumor registry reporting on clinicopathologic characteristics of Greek patients. BMC Endocr Disord, 16, 8.

Oh TG, Chung MJ, Park JY, et al (2012). Prognostic factors and characteristics of pancreatic neuroendocrine tumors: single center experience. Yonsei Med J, 53, 944-51.

Saglam S, Hacisahinogullari H, Ozturk N, et al (2015). Outcomes of first-line long-acting octreotide treatment in non-functional, advanced gastroenteropancreatic neuroendocrine tumors. $J B U O N, \mathbf{2 0}, 1201-5$.

Yao JC, Hassan M, Phan A, et al (2008). One hundred years after "carcinoid": epidemiology of and prognostic factors for neuroendocrine tumors in 35,825 cases in the United States. J Clin Oncol, 26, 3063-72.

This work is licensed under a Creative Commons AttributionNon Commercial 4.0 International License. 